INRA Prod. Anim.,

2007, 20 (3), 233-238

\title{
La vaccination chez les poissons d'élevage
}

\author{
C. QUENTEL ${ }^{1}$, M. BREMONT ${ }^{2}$, H. POULIQUEN ${ }^{3}$ \\ ${ }^{1}$ AFSSA, Site de Ploufragan/Brest, BP 70, F-29280 Plouzané, France \\ 2 INRA, UR892 Virologie et Immunologie Moléculaires, F-78352 Jouy-en-Josas, France \\ 3 INRA, Ecole Nationale Vétérinaire, UMR1035 Chimiothérapie Aquacole et Environnement, Atlanpole, \\ La Chantrerie, F-44307 Nantes, France \\ Courriel :c.quentel@brest.afssa.fr
}

$\mathrm{Au}$ cours des dernières décennies, l'industrie aquacole n'a cessé de se développer à travers le monde. En effet, la part des produits issus de l'aquaculture dans la production mondiale de poissons, mollusques et crustacés, qui représentait $25,8 \%$ de l'approvisionnement en 1998 a fortement progressé et a contribué en 2004 à $49 \%$ de l'approvisionnement du marché. L'extension des zones d'élevage étant relativement limitée, cette forte croissance s'accompagne d'une intensification des techniques d'élevage, et donc d'un accroissement des risques en particulier infectieux pouvant avoir un impact économique très important. Différents moyens de lutte contre les maladies existent :

- l'exclusion du pathogène basée sur la réglementation sanitaire : ceci est applicable uniquement dans des aires où la maladie n'est pas endémique, l'importation et le mouvement des animaux vivants et des œufs étant alors soumis à un contrôle sanitaire préalable. Dans ces zones indemnes, l'apparition d'une maladie peut être contrôlée grâce à l'abattage du stock d'animaux atteints et à une désinfection des bassins d'élevage ;

- la chimiothérapie grâce à l'utilisation de médicaments, notamment d'antibiotiques à des fins prophylactiques ou thérapeutiques : très efficace vis-àvis des bactéries, la chimiothérapie s'avère inopérante sur les virus. Ce type de traitements a longtemps été privilégié. Ce n'est plus le cas actuellement, du fait de l'apparition de l'antibiorésistance de nombreuses souches bactériennes mais également d'une prise de conscience des risques pour l'environnement et du souci grandissant pour la sécurité alimentaire ;

- la sélection génétique de souches résistantes à la (aux) maladie(s) : il peut s'agir d'une variabilité de la réponse spécifique à une maladie don- née ou bien relative à l'immunité non spécifique. A ce jour, cette approche reste expérimentale.

La vaccination apparaît comme un excellent moyen de prévenir et contrôler les maladies, en particulier là où elles sont endémiques. Dans ces zones, les populations de poissons sauvages agissent comme un réservoir d'infection inévitable pour les élevages. En effet, les survivants de nombreuses maladies restent porteurs sains asymptomatiques et constituent donc une menace permanente. De la même façon, des animaux vaccinés, résistants à la maladie, peuvent devenir des animaux porteurs du pathogène, cependant le risque n'est pas supérieur à celui que constitue les animaux sauvages. La vaccination, en particulier la vaccination de masse, a donc un rôle important dans le contrôle des maladies des poissons mais son utilisation doit tenir compte des considérations épidémiologiques de chaque maladie et de la nature du vaccin.

\section{1 / Le système immunitaire spécifique des poissons}

Les poissons, tout au moins les téléostéens, sont dotés d'un système immunitaire proche de celui des vertébrés supérieurs. Il est composé de deux systèmes majeurs, le système inné ou non spécifique et le système acquis, spécifíque du pathogène. Le premier est le plus ancien et est retrouvé à la fois chez les invertébrés et les vertébrés alors que le second est caractéristique des vertébrés. C'est ce dernier qui est sollicité lors d'une vaccination. La réponse immunitaire spécifique revêt deux formes, une réponse de type humoral avec la production anticorps et une réponse à médiation cellulaire, qui découlent de la stimulation respective des lympho- cytes $\mathrm{B}$ et $\mathrm{T}$ dont l'existence a été démontrée chez le poisson. La différentiation entre ces deux sous-populations lymphocytaires est réalisée par la mise en évidence de leurs marqueurs de surface dont les plus caractéristiques sont les immunoglobulines de surface chez les lymphocytes B (De Luca et al 1983) et le récepteur $\mathrm{T}$ chez les lymphocytes $\mathrm{T}$ (Partula et al 1995). L'existence de cellules présentatrices de l'antigène (Vallejo et al 1990, 1991) et des molécules du complexe majeur d'histocompatibilité (CMH) (Grimholt et al 2002, Hordvik et al 1993), qui jouent un rôle crucial dans la reconnaissance de l'antigène par les cellules $T$, a également été démontrée chez le poisson. A l'heure actuelle, de nombreuses avancées sont en cours en particulier concernant le clonage des cytokines des poissons et des gènes de leur récepteur. Ces molécules connues pour avoir des rôles clés dans la réponse immunitaire sont donc présentes chez les téléostéens et laissent à penser que la réponse immunitaire des poissons est soumise à des mécanismes régulateurs complexes comme chez les mammifères. Les poissons sont donc capables de reconnaître un antigène et de développer une réponse immunitaire spécifique. Il est donc possible de les vacciner. Cependant leur réponse est moins élaborée que celle des vertébrés supérieurs. Ainsi, ils ne produisent que deux classes d'immunoglobulines, des $\operatorname{IgM}$ et des IgD dont l'affinité est plus faible que celles des mammifères. De plus, la fonction de cette dernière classe est mal définie. Enfin, leur réponse mémoire est plus faible.

\section{2 / Voies d'administration des vaccins}

Les poissons peuvent être immunisés selon plusieurs voies, l'injection, la balnéation et l'administration orale. 
L'injection délivre à chaque animal une dose précise de vaccin. L'injection la plus généralement pratiquée est l'injection intra-péritonéale car elle apparaît comme la plus efficace (Ellis 1988). Elle peut être également intramusculaire ou sous-cutanée avec cependant un risque de provoquer des lésions et également de perte d'inoculum avant son absorption. Un adjuvant peut être combiné à la fraction vaccinale permettant d'augmenter la réponse immunitaire en stimulant l'activité immunitaire non spécifique. Ce mode de vaccination nécessite la manipulation individuelle des animaux avec souvent une anesthésie préalable, ce qui constitue des facteurs de stress pouvant affecter le système immunitaire. Par ailleurs, elle impose de lourdes charges de travail pour l'éleveur et n'est pas sans risque pour le manipulateur. Enfin, elle est délicate voire inapplicable chez les juvéniles et exige une grande quantité d'antigène.

La balnéation consiste à tremper le poisson dans une suspension vaccinale et peut être réalisée selon différentes procédures. Dans un premier temps, l'immersion hyper-osmotique a été proposée par Amend et Fender (1976). Une étude comparée de l'infiltration hyper-osmotique et de l'immersion directe a montré que la première est légèrement plus efficace pour des concentrations faibles en bactéries (Antipa et al 1980). Cependant, l'infiltration hyper-osmotique, étant, d'une part considérée comme un agent de stress, d'autre part à l'origine de dommages au niveau des branchies et de la peau, a été abandonnée tout au moins avec les antigènes particulaires. L'immersion directe est réalisée soit i) par immersion des poissons dans un petit bac contenant la suspension vaccinale diluée pendant quelques secondes avant de les remettre dans leur bassin d'élevage. La plupart des vaccins commerciaux sont prescrits par cette méthode, simple et rapide, bien qu'elle suppose la manipulation de l'ensemble du cheptel; ii) par addition du vaccin dans l'eau du bassin dont le niveau a été préalablement diminué minimisant ainsi les manipulations du poisson mais nécessitant une durée d'exposition plus longue à savoir une heure ou plus et une relative grande quantité de vaccin. Une variante à ces immunisations par immersion est la vaccination par spray qui exige, comme l'injection, la manipulation individuelle des animaux et n'est donc réalisable que sur de gros individus.
La vaccination par voie orale, avec l'antigène simplement mélangé à l'aliment, apparaît comme la méthode la plus facile pour une vaccination de masse des poissons, quelle que soit leur taille. Elle est rapide, ne demande que peu de travail à l'éleveur et évite tout agent de stress inhérent aux manipulations des poissons. Chez les téléostéens, l'intestin est capable d'adsorber des protéines bio-actives (Georgopoulou et al 1988) ainsi que des antigènes particulaires (Olafsen et Hansen 1992). A l'instar des mammifères, les poissons possèdent entre autres au niveau de l'intestin, un tissu lymphoïde associé aux muqueuses. Moins bien structuré que les plaques de Peyer des mammifères, ce tissu n'en est pas moins capable de réagir à l'ingestion d'antigène produisant ainsi une réponse immunitaire locale. Cette dernière est associée à une réponse immunitaire systémique dont la nature exacte est encore mal connue (Quentel et Vigneulle 1997). Cependant, l'efficacité et la durée de protection conférée à la suite d'une vaccination par voie orale sont très inférieures à celles observées avec les autres modes de vaccination. Une dégradation de l'antigène par les fluides gastriques se produit probablement, l'intubation anale s'avérant au moins, voire plus efficace que la balnéation et égale à l'inoculation (Johnson et Amend 1983). Enfin, la quantité d'antigène réellement ingéré par chaque individu n'est pas connue.

\section{3 / Les vaccins}

Un vaccin doit répondre à plusieurs critères comme ceux d'innocuité, d'efficacité et d'un coût de production raisonnable. Il ne doit pas provoquer d'effets secondaires, ne pas posséder une pathogénie résiduelle ni être susceptible de retrouver une certaine virulence. Il doit être immunogène, c'est-à-dire capable de stimuler les cellules du système immunitaire systémique et local en particulier contre les facteurs de virulence, et donc d'être protecteur visà-vis de la maladie considérée. Enfin, son coût incluant les efforts de recherche, les demandes d'autorisation de mise sur le marché et la production proprement dite, doit rester compatible avec la demande du marché. Ce dernier point est particulièrement crucial en aquaculture où le marché est relativement réduit par rapport à celui d'autres espèces animales et dans lequel les fabricants de vaccins hésitent à investir.

\section{1 / Les vaccins inactivés}

Ce sont pour l'essentiel des vaccins anti-bactériens produits par fermentation des bactéries suivie d'une inactivation au formol. Lors de la préparation du vaccin, le choix du ou des sérotype(s) utilisé(s) est primordial et peut varier selon la zone géographique à laquelle il est destiné et l'espèce cible (Toranzo et al 1997). Administrés par injection ou balnéation, ils s'avèrent très efficaces. Dans certains cas, ce type de formulation ne confère pas une protection suffisante et nécessite alors l'adjonction d'adjuvants pouvant entraîner l'apparition d'effets secondaires (Mydtling 1997). Des essais de vaccins à base de virus inactivés contre les principales maladies virales ont été testés. Bien qu'efficaces par injection, leur coût de production s'avère trop élevé pour en faire une stratégie économiquement viable. De plus, la plupart des maladies virales des poissons affectent les individus jeunes chez qui ce mode de vaccination à grande échelle est délicat voire impossible.

\section{2 / Vaccins atténués}

Les vaccins vivants atténués ont, dans un premier temps, été surtout étudiés vis-à-vis des maladies virales afin d'essayer de pallier la déficience des vaccins tués. La souche virale atténuée peut provenir d'un mutant non virulent ou être obtenue par passages successifs sur culture cellulaire. Ce type de vaccins mime donc une infection avec une réplication du virus dans les cellules de l'hôte réduisant ainsi la quantité d'antigène à délivrer. Ils peuvent être administrés par bain et sont donc peu stressants. Ils sont également connus pour stimuler la réponse immunitaire à médiation cellulaire responsable de l'élimination des micro-organismes intra-cellulaires (Kaufmann 1996) qu'ils soient d'origine virale ou bactérienne. Cependant, les vaccins atténués peuvent occasionnellement engendrer la maladie et la libération de particules vivantes, soit directement par l'eau, soit par les poissons vaccinés dans leur environnement, et ne sont donc pas compatibles avec les stratégies de contrôle à la fois vétérinaire et environnemental.

\section{3 / Les vaccins basés sur la technologie à ADN}

Avec le développement de la biologie moléculaire, de nouveaux types de vaccins ont vu le jour chez les animaux terrestres et sont en cours d'élaboration chez les poissons. Ainsi l'identification 
des antigènes protecteurs a permis l'élaboration de vaccins recombinants sub-unitaires constitués uniquement de ces antigènes. Ils sont produits in vitro par des techniques de génie génétique qui permettent la production d'une grande quantité d'antigène purifié à partir de systèmes capables d'exprimer des protéines hétérologues d'origine virale, bactérienne ou parasitaire. Intéressant en terme de coût de production et d'innocuité, ce type de vaccin est capable de fabriquer le squelette de la protéine ; cependant ces systèmes (colibacilles, cellules bactériennes, levures...) ne savent pas reproduire le repliement de la chaîne protéique et souvent échouent en terme de protection.

L'élaboration d'un système de génétique inverse pour la production de virus totalement non pathogènes par délétion d'un gène a été réalisé chez les poissons (Biacchesi et al 2000). De même l'immunisation génétique par simple inoculation intra-musculaire du gène $(\mathrm{ADN} n \mathrm{nu})$ a également permis d'obtenir des résultats prometteurs (Anderson et al 1996, Lorenzen et al 1998). Cependant, ces deux dernières constructions, qui miment bien l'action de l'agent pathogène en stimulant entre autres la réponse des cellules $\mathrm{T}$, posent un problème en terme de biosécurité du même ordre que les vaccins atténués traditionnels et peuvent être également considérés comme modifiant génétiquement l'hôte ce qui impose des exigences accrues au niveau de la réglementation pour une éventuelle commercialisation.

\section{4 / Vaccins contre les princi- pales maladies des poissons}

\section{1 / Les vaccins antibactériens}

A l'heure actuelle, il existe des vaccins pour une quinzaine de maladies bactériennes dans le monde. Beaucoup d'entre eux ont été développés de façon empirique et souvent encore à l'heure actuelle les antigènes protecteurs ne sont pas entièrement élucidés.

Les épizooties dues à des bactéries gram négatif sont les plus répandues et les premiers vaccins commerciaux ont été développés contre ces maladies. Il s'agit de vaccins contre les maladies bactériennes majeures : la yersiniose à Yersinia ruckeri qui sévit en eau douce chez les salmonidés et la vibriose à Listonella anguillarum (ex Vibrio anguillarum) présente essentiellement en aquaculture marine. Par la suite d'autres vaccins ont été développés contre les principaux sérotypes de $L$. anguillarum à l'origine de maladies chez le poisson et Vibrio ordalii. Ce dernier est souvent considéré comme le représentant du sérotype $\mathrm{O} 2$ de $L$. anguillarum dans les vaccins commerciaux. Or $V$. ordalii n'a jamais été identifié en Europe et ce type de vaccin apparaît donc peu approprié dans les pays européens. Ces vaccins sont composés de bactéries entières tuées au formol. Leur fabrication est facile et peu coûteuse. Administrés par injection ou par balnéation, ils s'avèrent très efficaces. D'autres vaccins de ce type ont été testés mais leur effet protecteur est souvent très variable. C'est le cas des vaccins contre la vibriose d'eau chaude à Vibrio $s p$ ou encore du vaccin contre la vibriose d'eau froide due à Vibrio salmonicida pour lequel l'adjonction d'adjuvant permet d'augmenter l'immunité. $\mathrm{Au}$ cours des années 80 , un vaccin contre la furonculose à base Aeromonas salmonicida tués additionnés d'adjuvant huileux s'est avéré très efficace en terme de niveau et durée de protection chez le saumon atlantique (Salmo salar). Néanmoins, la présence de lésions granulomateuses adhérant aux viscères chez les animaux vaccinés les rend invendables et le vaccin ne fut pas adopté par les éleveurs. Il semblerait que la production d'A. salomonici$d a$ dans des conditions restrictives en fer pourrait permettre d'augmenter l'immunogénicité du vaccin. Des études portant sur des vaccins vivants ont montré pouvoir stimuler l'immunité à médiation cellulaire (Vaughan et al 1993, Marsden et al 1996). Or, cette dernière joue un rôle important dans l'acquisition de l'immunité vis-à-vis des microorganismes intracellulaires comme $A$. salmonicida. Il a également été montré que l'immunité protectrice est améliorée quand $A$. salmonicida est associé à $V$. salmonicida démontrant une protection croisée (Hoel et al 1998). Pour l'ensemble de ces bactéries, des vaccins monovalents commerciaux existent, cependant la tendance actuelle est la proposition de vaccins multivalents composés soit de plusieurs sérotypes d'un même genre, soit de plusieurs genres bactériens. La multiplication des valences dans la formulation vaccinale ne semble pas altérer leur immunogénicité. La plupart ont une autorisation au niveau national dans les nombreux pays où les maladies sévissent et de plus en plus de demandes de reconnaissance mutuelle sont présentées. D'autres vaccins sont commercialisés mais avec une distribution moins large. Un vaccin, constitué d'antigènes cellulaires et solubles, contre la pasteurellose due à Photobacterium damselae (ex Pasteurella piscicida) à l'origine de forte mortalité chez le bar (Dicentrarchus labrax) et la daurade (Sparus aurata) est actuellement commercialisé dans les pays méditerranéens. Un rappel est cependant nécessaire pour obtenir une bonne protection. Aux Etats-Unis, la vaccination contre la septicémie entérique du poisson-chat, Ictalarus punctatus, à Edwardsiella ictalari à l'aide de vaccins tués ou vivants (Klesius et Shoemaker 1999) est autorisée. Leur efficacité démontrée au laboratoire n'a cependant pas été totalement retrouvée sur le terrain. Un vaccin sub-unitaire contre Aeromonas hydrophila est utilisé en Russie chez la carpe.

Les septicémies causées par des bactéries gram positif, comme la streptococcose due à Streptococcus iniae et l'enterococcose à Strepococcus iniae, entraînent de fortes mortalités en été chez certaines espèces de poisson. Quelques pays où ces deux bactéries posent de graves problèmes ont autorisé des vaccins inactivés qui sont administrés par injection et dont l'effet protecteur est relativement bon.

La corynébactériose, due à Renibacterium salmoninarum qui est un bacille gram positif très difficile à cultiver in vitro, provoque chez les salmonidés une maladie chronique. Seuls le Canada, les Etats-Unis et le Chili autorisent la vaccination contre cette maladie avec un vaccin vivant d'Arthrobacter sp. Un vaccin à $\mathrm{ADN}$ a également été testé vis-à-vis de cette maladie (Gomez-Chiarri et al 1996).

La piscirickettsiose à Piscirickettsia salmonis, pathogène intracellulaire obligatoire, constitue un problème grave au Chili pour les élevages de saumon coho (Oncorhynchus kisutch). Un vaccin inactivé injectable est autorisé dans ce pays, cependant son pouvoir protecteur est faible. A l'heure actuelle, des vaccins recombinants contre l'antigène OspA de $P$. salmonis sont à l'étude (Kuzyk et al 2001) et s'avèrent efficaces dans les conditions de laboratoire.

\section{2 / Les vaccins antiviraux}

En dépit des nombreuses recherches menées aussi bien par l'industrie pharmaceutique que par les organismes de recherche, très peu de vaccins antiviraux ont une autorisation de mise sur le marché. Tous sont des vaccins résultant 
de l'inactivation du virus ou de protéines recombinantes. Ils peuvent être formulés avec des adjuvants huileux ou être un composant de vaccins multivalents. Aucun vaccin vivant atténué ou à ADN n'a obtenu d'autorisation pour des raisons de biosécurité et/ou de réglementation. Cependant un vaccin à ADN contre la Nécrose Pancréatique Infectieuse (NPI) a fait l'objet d'essais cliniques au Canada.

Concernant la NPI due à un aquabirnavirus affectant les salmonidés, des vaccins sont commercialisés en Norvège et au Chili et depuis très récemment en Grande Bretagne. Ce sont des vaccins multivalents ou un vaccin recombinant avec la protéine VP2 exprimée par Escherichia coli associée à des antigènes bactériens. Leur efficacité en conditions expérimentales n'est pas retrouvée sur le terrain, peut-être du fait de l'omniprésence du virus dans les élevages.

Concernant les rhabdoviroses chez le poisson, à savoir la Nécrose Hématopoiétique Infectieuse (NHI), la Septicémie Hémorragique Virale (SHV) et la Virémie Printanière de la Carpe (VPC) de nombreux travaux utilisant les approches classiques (vaccins inactivés ou atténués) ont été effectués au cours des dernières décennies mais n'ont pas permis d'aboutir à une commercialisation de vaccins. La réplication du virus de la NHI chez la truite est illustrée sur la figure 1. Les nombreux essais réalisés avec des vaccins recombinants sub-unitaires basés sur la glycoprotéine stimulant la production d'anticorps neutralisants se sont avérés insuffisants en terme de protection. Par contre de bons résultats ont été obtenus avec des vaccins à $\mathrm{ADN}$ vis-à-vis de la NHI et la SHV (Anderson et al 1996, Lorensen et al 1998, Boudinot et al 1998). Une autre approche, le système de génétique inverse, semble prometteuse (Biacchesi et al 2000).

L'étiologie virale de la Maladie du Sommeil (MS) chez la truite arc-en-ciel en eau douce et la Maladie du Pancréas (MP) chez le saumon atlantique (Salmo salar) en mer a récemment été mise en évidence (Castric et al 1997, Rolwey et al 1998). Dans les deux cas il s'agit de virus appartenant à la famille des alphavirus aquatiques. Un vaccin inactivé contre la MP permettant une réduction des lésions histopathologiques a été testé en Irlande et Norvège. Une autre approche proposant l'obtention d'un ADN complémentaire (ou ADNc) infectieux non pathogène obtenu à par- tir de l'ADN viral de la MS vient d'être réalisée. Les juvéniles infectés par immersion par le virus recombinant apparaissent ensuite protégés vis-à-vis du virus sauvage (Moriette et al 2006).

Un autre virus appartenant à la famille des Orthomyxoviridae, le virus de l'Anémie Infectieuse du Saumon (AIS), est à l'origine de forte perte économique dans les élevages de saumons en Norvège et en Ecosse mais aussi en Amérique du nord. Des vaccins commerciaux sont disponibles sur le continent américain mais pas en Europe. Il s'agit de vaccins inactivés souvent multivalents dont les effets semblent très variables sur le terrain. Des recherches utilisant les technologies d'ADN recombinant sont à l'heure actuelle en cours.

Figure 1. Truitelles infectées par un virus recombinant vNHI-LUC exprimant la Luciférase. Les truitelles vivantes sont observées post-infection aux temps indiqués grâce à un système d'imagerie (IVIS Xenogen) permettant de visualiser in vivo la réplication du virus chez les truites infectées. On remarque qu'aux temps très précoces, la réplication virale est localisée à la base des nageoires représentant la «porte d'entrée» du virus.

4 jours

$40 \mathrm{~h}$

$24 \mathrm{~h}$

$16 \mathrm{~h}$

$8 \mathrm{~h}$

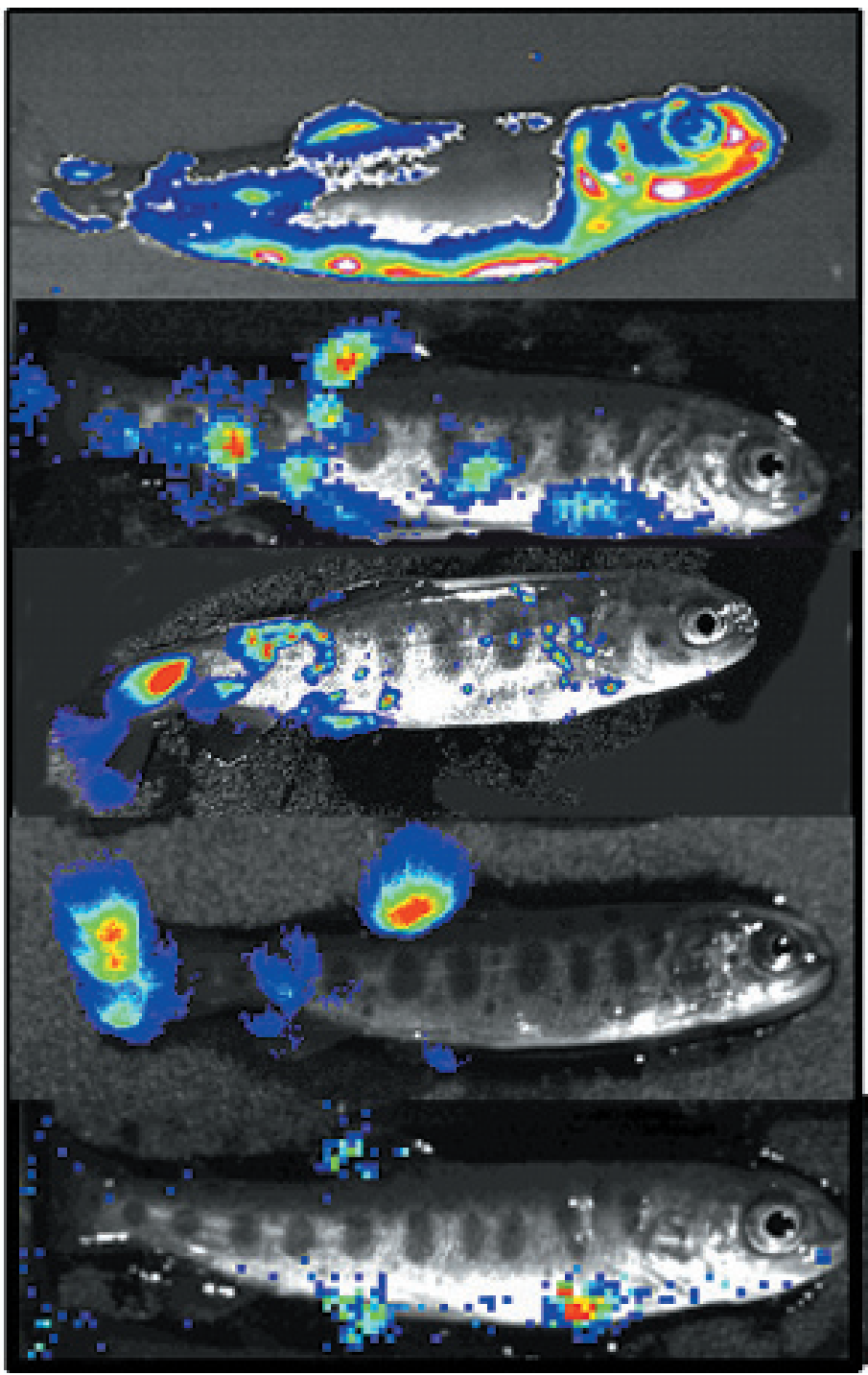


Initialement destinés essentiellement aux salmonidés, la vaccination bactérienne s'est progressivement étendue à d'autres espèces d'élevage. Très peu de vaccins antiviraux sont commercialisés et il n'existe pas de vaccins antiparasitaires.

Une meilleure connaissance de la réponse immunitaire des différentes espèces de poissons en particulier de la réponse à médiation cellulaire et de l'immunité locale associée à une identification précise des antigènes protecteurs devrait permettre d'optimiser la vaccinologie en aquaculture. Par ailleurs, les résultats prometteurs obtenus avec la vaccination à $\mathrm{ADN}$ devraient permettre à moyen terme le développement de futurs vaccins destinés aux poissons.

Enfin, il faut évoquer la possibilité de préparer des autovaccins à usage vétérinaire, c'est-à-dire des médicaments vétérinaires immunologiques destinés à provoquer une immunité active à partir d'organismes pathogènes provenant d'un ou de poisson(s) d'un même élevage, inactivés et utilisés pour le traite- ment des poissons de cet élevage. Néanmoins, la préparation de ces autovaccins est aujourd'hui limitée car elle doit être effectuée par une personne qualifiée, ayant obtenu une autorisation délivrée par l'Agence Française de Sécurité Sanitaire des Aliments (AFSSA) (décret $\mathrm{n}^{\circ}$ 2005-374 du 20 avril 2005 paru au J.O. du 23 avril 2005). Y sont astreints tous les laboratoires, de statut privé ou public, y compris les laboratoires départementaux vétérinaires recevant les prélèvements qu'ils analysent.

\section{Références}

Amend D.F., Fender D.C., 1976. Uptake of bovine serum albumin by rainbow trout from hyperosmotic: a model for vaccinating fish Science, 192, 793-794.

Anderson E.D., Mourich D.V., Fahrenkrug S.C., LaPatra S., Shepherd J., Leong J.A., 1996. Genetic immunization of rainbow trout (Oncorhynchus mykiss) against infectious hematopoietic necrosis virus. Mol. Mar. Biol. Biotechnol., 5, 114-122.

Antipa R., Gould R., Amend D.F., 1980. Vibrio anguillarum vaccination of sockeye salmon, Oncorhynchus nerka (Walbaum) by direct and hyperosmotic immersion. J. Fish Dis., 3, 161-165.

Biacchesi S., Thoulouse M.I., Bearzotti M., Yu Y.X., Bremont M., 2000. Recoveryof NV knockout infectious hematopoietic necrosis virus expressing foreign genes. J. Virol., 74, $11247-$ 11253.

Boudinot P., Blanco M., de Kinklin P., Benmansour A., 1998. Combined DNA immunization with the glycoprotein gene of viral hemorrhagic septicemia virus and infectious hematopoietic necrosis virus induces doublespecific protective imity and nonspecific response in rainbow trout. Virology, 249, 297306.

Castric J., Baudin Laurencin F., Brémont M., Jeffroy J., Le Ven A., Bearzotti M., 1997. Isolation of the virus responsible for sleeping disease in experimentally infected rainbow trout (Oncorhynchus mykiss). Bull. Eur. Assoc. Fish Pathol., 17, 27-30.

De Luca D., Wilson M., Warr G.W., 1983. Lymphocytes heterogeneity in the trout, Salmo gairdeneri, defined with monoclonal antibodies to IgM. Eur. J. Immunol., 13, 546-551.

Ellis A.E., 1988. General principles of fish vaccination. In: Ellis A.E. (Eds), Fish Vaccination, Academic Press, London, UK, 1-19.

Georgopoulou U., Dabrowski K., Sire M.F., Vernier J.M., 1988. Absorption of intact proteins by the intestinal epithelium of trout, Salmo gairdneri. A luminescence enzyme immunoassay and cytochemical study. Cell Tissue Res., $251,145-152$

Gomez-Chiarri M., Brown L.L., Levine R.P., 1996. Protection against Renibacterium salmoni- narum infection by DNA-based immunization. Int. Cong. Biol. Fishes, San Francisco State University, USA, 155-157.

Grimholt U., Drablos F., Jorgensen S.M., Hoyheim B., Stet R.J.M., 2002. The major histocompatibility class I locus in Atlantic salmon (Salmo salar): polymorphism, linkage analysis and protein modelling. Immunogenetics, 54, $570-581$

Hoel K., Reitan L.J., Lillehaug A., 1998. Immunological cros-reactions between Aeromonas salmonicida and Vibrio salmonicida in Atlantic salmon (Salmo salar L.) and rabbit. Fish Shellfish Immunol., 8, 171-182.

Hordvik I., Grimholt U., Fosse V.M., Lie O., Endresen C., 1993. Cloning and sequence analysis of cDNAs encoding the MHC class II B chain in Atlantic salmon (Salmo salar) Immunogenetics, 37, 437-441.

Johnson K.A., Amend D.F., 1983. Efficacy of Vibrio anguillarum and Yersinia ruckeri bacterins applied by oral and anal intubation of salmonids. J. Fish Dis., 6, 473-476.

Kaufmann S.H.E., 1995. Immunity to intracellular microbial pathogens. Immunol. Today, 16 , 338-342.

Klesius P.H., Shoemaker C.A., 1999. Development and use of modified live Edwardsiella ictaluri vaccine against enteric septicemia of catfish. Adv. Vet. Med., 41, 523537.

Kuzyk M.A., Burian J., Machander D., Dolhaine D., Cameron S., Thornthon J.C., Kay W.W., 2001. An efficacious recombinant subunit vaccine against the salmonid rickettsial pathogen Piscirickettsia salmonis. Vaccine, 19, 23372344.

Lorenzen N., Lorenzen E., Einer-Jensen K., Heppell J., Davis H.L., 1988. Protective immunity to VHS in rainbow trout (Oncorhynchus mykiss, Walbaum) following DNA vaccination. Fish Shellfish Immunol., 8, 261-270.

Marsden M.J., Vaugham L.M., Foster T.J., Secombes C.J., 1996. A live ( $\Delta$ aroa) Aeromonas salmonicida vaccine for furonculosis preferentially stimulates $\mathrm{T}$ cell responses relative to $\mathrm{B}$ cell responses in rainbow trout (Oncorhynchus mykiss). Infect. Immun., 64, 3863-3869.
Midtling P.J., 1997. Vaccinated fish welfare: protection versus side-effects. In: Gudding $\mathrm{R}$, Lillehaug A., Midtling P.J., Brown F. (Eds), Dev. Biol. Stand., Basel, Karger. Fish Vaccinol., 90, 371-379.

Moriette C., LeBerre M., Lamoureux A., Lai T.H., Brémont M., 2006. Recovery of a recombinant salmonid alphavirus fully attenuated and protectrice for rainbow trout. J. Virol., 80, 40884098.

Olfasen J.A., Hansen G.H., 1992. Intact antigen uptake in intestinal marine fish larvae. J. Fish Biol., 40, 141-156.

Partula S., de Guerra A., Fellah J.S., Charlemagne J., 1995. Structure and diversity of the $T$ cell antigen receptor beta-chain in a teleostéen fish. J. Immunol., 155, 699-706.

Quentel C., Vigneulle M., 1997. Antigen uptake and immune responses after oral vaccination. In: Gudding R., Lillehaug A., Midtling P.J., Brown F. (Eds), Dev. Biol. Stand., Basel, Karger. Fish Vaccinol., 90, 69-78.

Rowley H.M., Doherty C.E., McLoughin M.F., Welsh M.D., 1998. Isolation of salmon pancreas disease virus (SPDV) from farmed Atlantic salmon, Salmo salar L. J. Fish Dis., 21, 469-471.

Toranzo A.E., Santos Y., Barja J.L., 1997. Immunization with bacterial antigens: Vibrio infections. In: Gudding R., Lillehaug A., Midtling P.J., Brown F. (Eds), Dev. Biol. Stand., Basel, Karger. Fish Vaccinol., 90, 93-105.

Vallejo A.N., Miller N.W., Jorgensen T., Clem L.W., 1990. Phyllogeny of immune recognition: antigen processing/presentation in channel catfish immune responses to hemocyanins. Cell. Immunol., 130, 362-377.

Vallejo A.N., Miller N.W., Clem L.W., 1991. Phylogeny of immune recognition: processing and presentation of structurally defined proteins in channel catfish immune responses. Develop. Immunol., 1, 137-148.

Vaughan L.M., Smith P.R., Foster T.J., 1993. An aromatic-dependant mutant of the fish pathogen Aeromonas salmonicida is attenuated in fish and is effective as live against the the salmonid disease furonculosis. Infect. Immun. 61, 2172-2181. 


\title{
Résumé
}

Les poissons, tout au moins les téléostéens, sont dotés d'un système immunitaire proche de celui des vertébrés supérieurs. Il est composé de deux systèmes majeurs, le système inné ou non spécifique et le système acquis, spécifique du pathogène. Les poissons sont capables de reconnaître un antigène et de développer une réponse immunitaire spécifique. Il est donc possible de les vacciner. Les poissons peuvent être immunisés selon plusieurs voies, l'injection, la balnéation et l'administration orale. Différents types de vaccins existent : des vaccins inactivés, qui sont pour l'essentiel des vaccins anti-bactériens produits par fermentation des bactéries suivie d'une inactivation au formol, des vaccins vivants atténués qui, dans un premier temps, ont été surtout étudiés vis-à-vis des maladies virales afin d'essayer de pallier la déficience des vaccins tués. Bien qu'efficaces par balnéation, ils ne sont pas commercialisés car incompatibles avec les stratégies de contrôle à la fois vétérinaire et environnemental. Enfin, avec le développement de la biologie moléculaire de nouveaux types de vaccins sont en cours d'élaboration chez les poissons comme les vaccins recombinants sub-unitaires dont l'efficacité n'a cependant pas été démontrée. Par contre, les résultats avec les vaccins à $\mathrm{ADN}$ sont très prometteurs et ces derniers devraient dans un avenir proche permettre d'optimiser la vaccination chez les poissons.

\begin{abstract}
Farm fish vaccination

Fish, at least teleosts, are equipped with an immune system similar to that of terrestrial vertebrates. It is divided into two main systems, the innate or non-specific system and the adaptive system, specific to the pathogen. Fish are able to recognize an antigen and to develop a specific immune response. Thus, it is possible to vaccinate them. Fish can be immunized using several ways including injection, balneation and oral administration. Various types of vaccines exist : inactivated vaccines which are essentially anti-bacterial vaccines produced by bacteria fermentation followed by an inactivation with formalin, and live attenuated vaccines, which were initially especially studied with respect to viral diseases in order to try to compensate for the deficiency of the killed vaccines. Although effective by balneation, they are not marketed because they are not compatible with veterinary and environmental control strategies. Lastly, with the development of molecular biology, new types of vaccines are under development in fish like the recombinant subunit vaccines whose effectiveness has, however, not been shown. On the contrary, the results with DNA vaccines are very promising and the latter should, in a near future, optimize fish vaccination.
\end{abstract}

QUENTEL C., BREMONT M., POULIQUEN H., 2007. La vaccination chez les poissons d'élevage. INRA Prod. Anim., $20,233-238$. 